\title{
Etude de la périodicité de croissance d'après les plans isodynamiques des os
}

\author{
par
}

W. CLERC

$\mathrm{D}^{\mathrm{r}}$ ès-Sciences

Sverdlovsk (Ekatherinbourg).

Avec 16 figures dans le texte.

La méthode que je soumets à la critique des zoologistes fut énoncée pour le première fois en 1916 uniquement au point de vue de la définition de l'âge chez les Poissons (1916a). Peu après, j'en ai donné une interprétation théorique $(1916 b)$. Le même thème, considéré sous d'autres points de vue, fut le sujet de mes communications au premier et au deuxième Congrès des zoologistes russes $(1923,1924)$.

Sous le nom de "méthode des coupes transversales d'os massifs" ma méthode a été adoptée en Russie, plus spécialement pour l'étude des Acipenseridae. Comme elle peut être appliquée avec succès à l'étude de la périodicité de croissance chez tous les Vertébrés en général, je crois utile de la mettre à la portée de tous ceux qui s'intéressent à cette question.

La structure des os au point de vue de l'analyse de la croissance, a été étudiée presque exclusivement chez les Poissons; c'est pourquoi je m'adresserai principalement à ce groupe au cours de mes démonstrations.

Depuis longtemps les ichtyologues s'occupent de la détermination de l'âge des Poissons, ceci dans un but pratique. Ces derniers utilisent des procédés applicables en gros tout en apportant des résultats approximatifs. Il en résulte que le défaut général des méthodes usitées en ichtyologie pratique est de ne pas satisfaire 
aux exigences d'exactitude nécessaires pour des recherches qui nous intéressent.

Les imperfections de ces méthodes m'ayant poussé à l'élaboration d'un nouveau procédé d'étude, j'exposerai brièvement la critique de celles-ci.

L'âge du Poisson est ordinairement déterminé par l'étude de l'écaille, des os plats, des vertèbres, ou des otolithes; plus récemment, on utilisa en Russie la méthode que nous exposons.

L'étude de l'écaille se réduit à l'examen des zones vues par transparence, des stries concentriques de la surface; ou, enfin, de l'un et l'autre simultanément.

La densité relative de la répartition des zones claires, des stries, et surtout les infractions à la régularité de leur répartition, sont considérées comme coüncidant avec des variations dans la vitesse de croissance. Il est admis que les zones plus claires correspondent à un ralentissement de croissance occasionné soit par l'hivernage, soit par la maturation des produits sexuels.

Si personnelle que puisse être l'évaluation de ces "couches annuelles ), les résultats obtenus sont utilisables dans l'ichtyologie pratique, à condition que les Poissons étudiés appartiennent à des troupes d'individus réunis, soit pour le frai, soit pour d'autres migrations. Ces troupes étant, pour des raisons écologiques, homogènes quant à leur âge et à leur race, malgré les erreurs de calcul une comparaison sommaire est possible. Pour des recherches d'ordre scientifique, comme, par exemple, l'étude de différentes phases de la croissance, l'analyse de l'écaille donne, à elle seule, des résultats peu probants.

Les observateurs ne discernent pas toujours nettement la limite des zones claires. Souvent les striations n'ont pas d'irrégularités bien marquées, et cela justement dans les régions de l'écaille où ces deux indications peuvent être juxtaposées: voici donc une source d'erreur possible.

L'absence de précision dans les données fournies par l'écaille provient aussi de la structure. L'écaille se compose de lamelles imbriquées de substance osteoïde; chaque écaille ayant la forme d'un cône plus ou moins haut. La croissance de l'écaille est sensiblement unilatérale. Ainsi l'écaille prise sur un Poisson, même vivant, ne peut pas donner un tableau bien net de la croissance. Mais il est rare que l'on puisse opérer avec de l'écaille absolument fraîche. 
L'écaille est composée de couches très minces qui sont loin d'être homogènes, puisque les réactifs (par exemple le bleu de méthyle) les colorent d'une manière différente.

C'est pour cette raison que les diverses couches de chaque écaille se comportent différemment pendant la dessication. Il s'en suit une dislocation des couches qui modifie complètement les propriétés optiques de l'écaille.

L'écaille étudiée d'après le procédé usuel peut donc servir, quand il s'agit de recherches spéciales sur la périodicité de croissance, tout au plus à un triage sommaire.

Bien des travaux concernant le groupement des Poissons d'après leur âge, se basent sur l'analyse des os plats. J'ai donné, en 1916, une critique assez détaillée de cette méthode (1916a). Mes recherches postérieures ont confirmé mes conclusions d'alors. Les objections faites au sujet de l'écaille s'appliquent également aux os plats. Il en résulte que les propriétés optiques sur lesquelles se base l'analyse des couches annuelles dépendent grandement $d u$ mode de fixation et de conservation de l'os. Les os plats ont de plus l'inconvénient d'être sujets à une résorption de la substance osseuse aussi bien à l'extérieur qu'à l'intérieur.

Sur les trois figures représentées cidessous nous voyons les cas les plus fréquents de la répartition des couches dans les os plats.

La fig. 1 représente un cas assez rare où les couches périostales sont d'égale épaisseur sur les deux faces de l'os. Les rayons de lumière forment

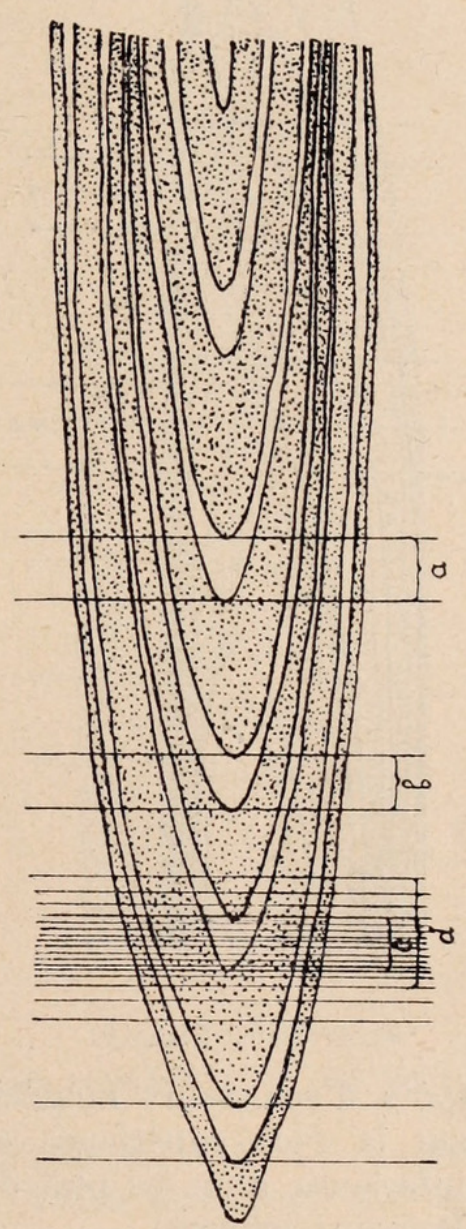

FIG. 1.

Schéma des dépôts périostaux uniformes d'un os plat de Poisson.

$a, b, c=$ limites réelles des zones claires; $d=$ largeur apparente d'une zone. sur le bord des couches transparentes $(a, b)$ des raies claires dont la largeur permet d'évaluer l'épaisseur relative des couches en question. 
La fig. 2 représente un cas bien plus fréquent. Les couches sont plus minces sur la surface inférieure que sur la surface supérieure de l'os plat. Il est évident que le faisceau de rayons lumineux

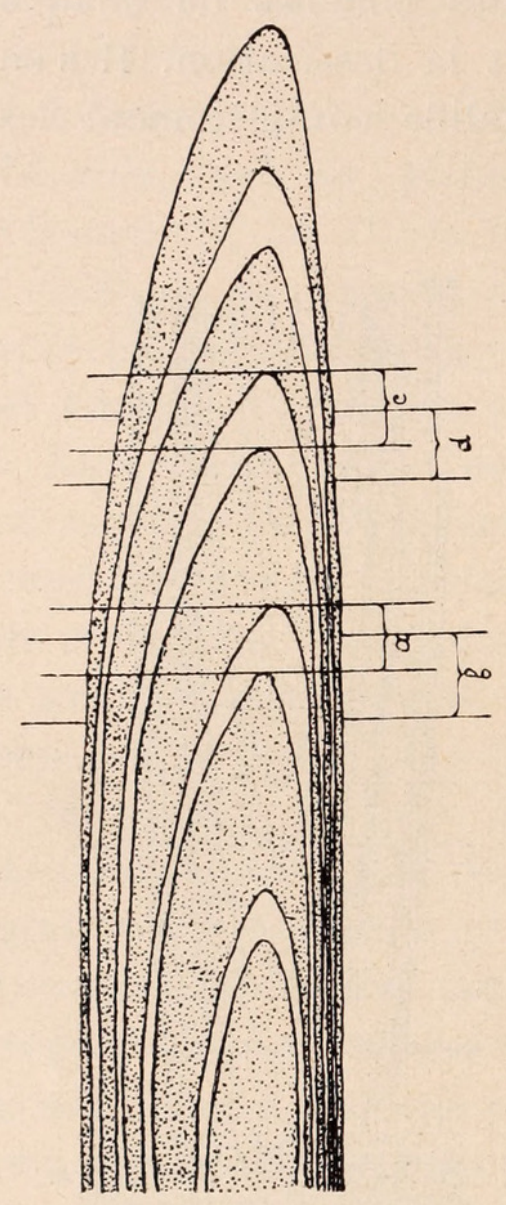

FIG. 2.

Dépôts d'épaisseurs inégales sur la face supérieure et inférieure d'un os plat de Poisson (schéma). donnera, en traversant la courbure des couches claires - courbure qui est ici plus compliquée, - une image $(a-b ; c-d)$ moins nette que dans le cas précédent.

Chaque zone, que l'on considère comme étant le dépôt d'une année entière, est composée d'un certain nombre de couches plus minces. Lors de la dessication ou de la déshydratation, ces couches se déformant inégalement, on comprend facilement que l'os éclairé en lumière directe ou transmise, donnera lieu à des appréciations très contradictoires. Une analyse sérieuse devient impossible dans ces conditions.

La fig. 3 représente un déplacement apparent de la substance osseuse vers le bord massif de l'os plat. A la courbure mentionnée plus haut s'ajoute sur la surface inférieure une seconde courbure qui est suivie d'un si brusque amincissement des couches qu'elles paraissent interrompues. Très souvent on observe simultanément une résorption superficielle. Il est clair que le tableau que nous offrira l'os dans ces conditions ne correspondra pas au groupement réel des dépôts du périoste.

La résorption interne joue également un rôle dans l'analyse des os plats, car elle envahit des séries entières de couches annuelles, ceci surtout chez les Poissons âgés.

Le relief des faces des os plats est souvent très accidenté. Dans ce cas, l'accroissement périostal qui se conforme au relief, donne, vu par transparence, un tableau fort compliqué. Pour peu qu'une résorption interne, même insignifiante, ait lieu, l'analyse de l'os devient complètement impossible. 
La lecture de l'âge des Poissons d'après les vertèbres rend souvent les observateurs très perplexes. Il arrive que des personnes très expérimentées tirent des conclusions toutes différentes de l'analyse du même matériel.

Bien des causes concourent à cette incertitude. D'une manière générale, les dépôts ne sont bien visibles que s'ils atteignent une certaine épaisseur. Or, dans les vertèbres, ces dépôts sont très inégaux. Sur la surface amphicœle, les dépôts sont si insignifiants que les couches deviennent indistinctes. Quoique l'épaisseur des dépôts soit suffisante, dans la direction radiale, la forte courbure des couches gène l'examen, car le tableau qu'elles présentent varie avec les changements de l'éclairage.

La présence, à la périphérie de la vertèbre, d'une grande quantité de fortes fibres collagènes, concourt à rendre peu nettes les limites des différentes couches.

Lors de la dessication, les couches minces étant hétérogènes se disloquent au niveau des courbures, ces dislocations en se prolongeant sur la surface amphicoele se manifestent par des fissures béantes.

La résorption interne, très intense dans les vertèbres, envahit les dépôts périostaux à différents degrés, ce qui rend l'analyse de la croissance encore moins certaine.

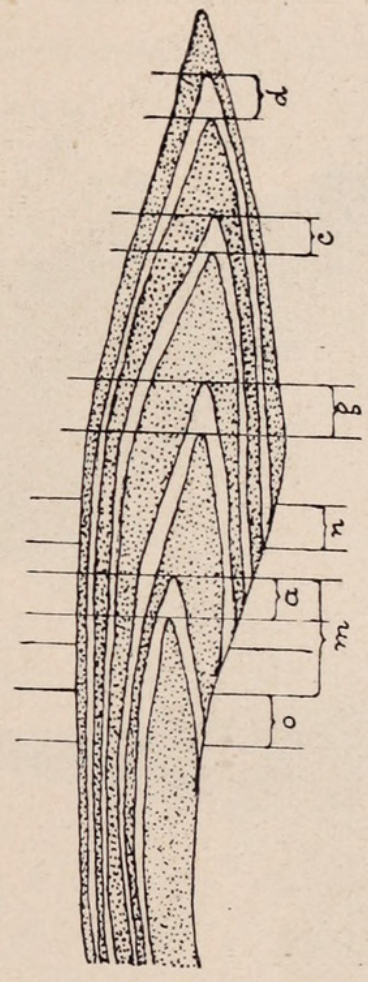

FIG. 3

Double courbure

des couches dans un os plat.

(Schéma.) $a, b, c, d=$ largeur réelle des zones claires; $a$, $n=$ zone claire d'apparence complexe; $m=$ zone claire d'apparence complexe.

Les fig. 4, 5, 6 font voir la disposition des dépôts périostaux ${ }^{1}$.

On détermine quelquefois l'âge des Poissons d'après les couches concentriques des otolithes. Cette méthode, malgré sa grande

1 Les microphotographies 4, 5, 6, 7, 8, 12, 13, 15 et 16 représentent des coupes obtenues par polissage au moyen de poudre fine de pierre ponce et d'une huile minérale. 
valeur, est moins répandue qu'elle ne le mérite. Cela tient probablement au fait que les études théoriques sur la croissance des Poissons sont de date relativement récente. Quant à l'utilisation des otolithes pour l'ichtyologie pratique, les difficultés très réelles de leur extraction chez les Poissons de petite taille, leur exiguïté chez ces derniers, jointe à la difficulté de l'emploi du microtome,

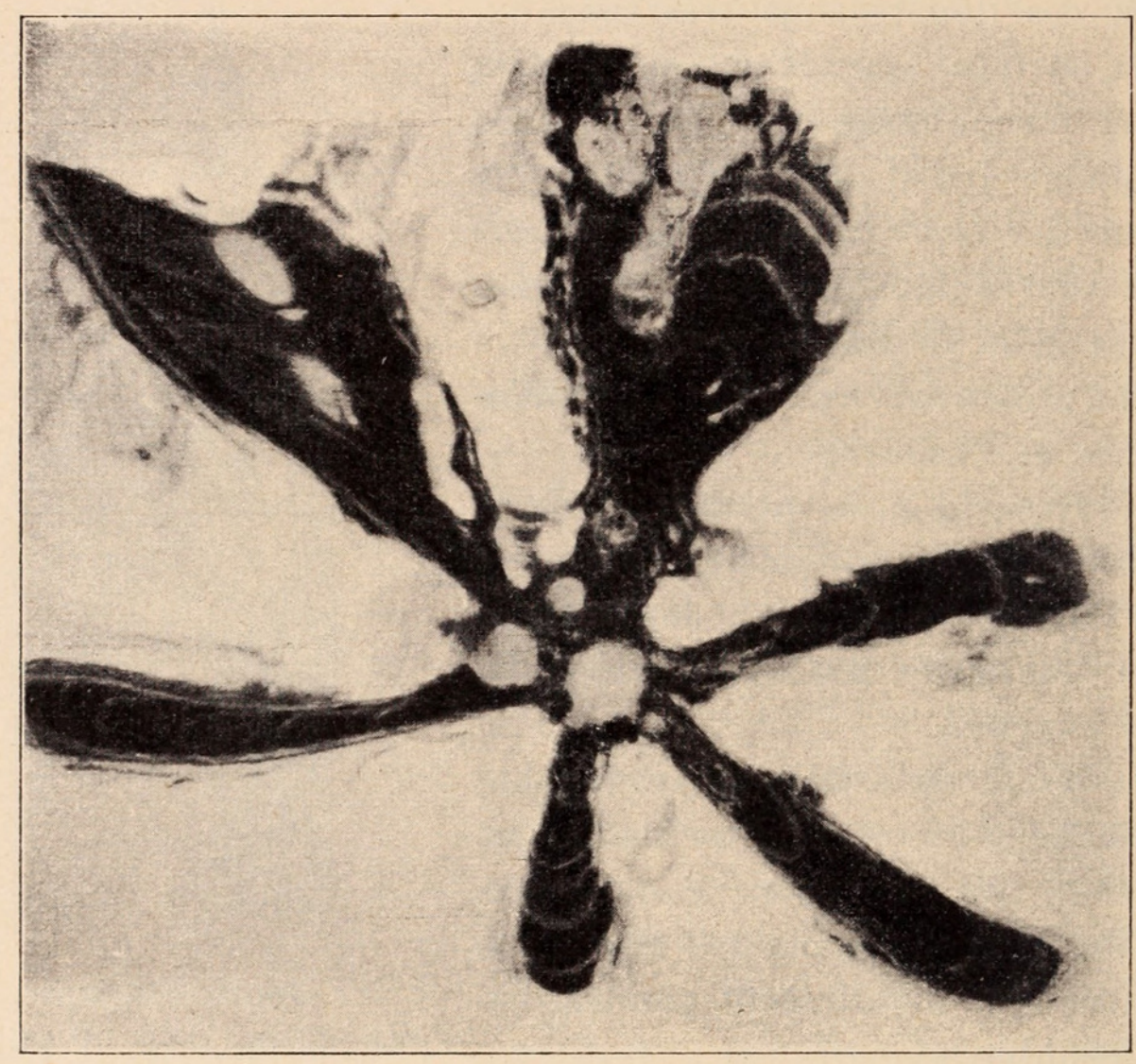

Fig. 4.

Vertèbre du Carassin, coupe transversale.

s'y opposent. Ces objections subsistent, d'ailleurs, quand il s'agit des recherches qui nous intéressent.

J'ai pu constater les imperfections de ces diverses méthodes en étudiant, chez les Poissons, des phases d'une durée moindre que le cycle annuel, ou, au contraire, des périodes d'une série d'années.

L'ichtyologie a trop simplifié le problème de la croissance en admettant que celle-ci coïncide complètement avec les change- 
ments de saisons. C'était exagérer l'influence du milieu, du climat et d'autres causes externes, tandis qu'en réalité le processus de croissance est un phénomène complexe qui dépend surtout de causes internes et se répercute jusque dans la structure intime de l'os. Il est vrai que certains auteurs indiquent la présence de couches correspondant aux périodes de frai, mais, dans ce cas encore, il y a tendance à simplifier la question.

Il a donc fallu mettre de côté les méthodes créées pour les besoins de l'ichtyologie pratique, et recourir à d'autres procédés permettant de faire une analyse histologique plus complète des os.

Les histologistes ont, en général, peu étudié les os du point de vue qui nous intéresse. Aussi, ne trouvonsnous dans la littérature de cette question que des données éparses.

C'est chez Loviaguine (1915) que j'ai trouvé des données répondant le mieux au point de vue qui nous intéresse. Loviaguine indique qu'il a constaté dans une partie du fémur humain des traces de dépôt des principales lamelles externes, traces qui avaient persisté jusqu'à l'âge de 30 ans.

Gebhard t (1900-05) a trouvé dans le squelette de nombreuses espèces de Mammifères, des régions osseuses montrant des séries de la-

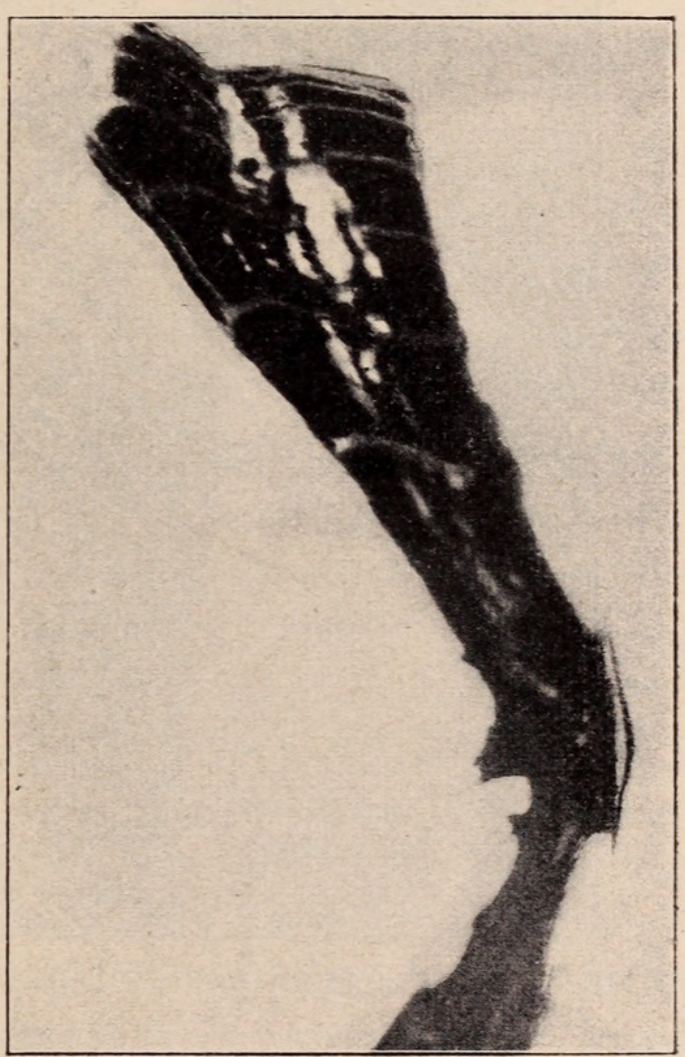

FIG. 5 .

Partie d'une coupe radiale d'une vertèbre de Carassin. melles basales bien distinctes, aussi bien internes qu'externes. Chez ce même auteur, j'ai trouvé des indications précieuses qui m'ont conduit à la découverte d'une méthode pratique de lecture des phases de croissance. Gebhardt indique la présence dans les os, de régions où la superposition de lamelles externes est bien nette, cela même chez 
des animaux dont les os sont très spécialisés dans leur structure et leurs fonctions mécaniques (le Phoque, la Baleine, la Musaraigne, le Fourmilier, etc.). C'est en vérifiant la remarque de GebhardT sur des os de différents animaux, plus spécialement sur une espèce de Tatou, sur la Taupe, et, enfin, sur l'Homme, que je fus amené à mon idée de l'utilisation des plans isodynamiques.

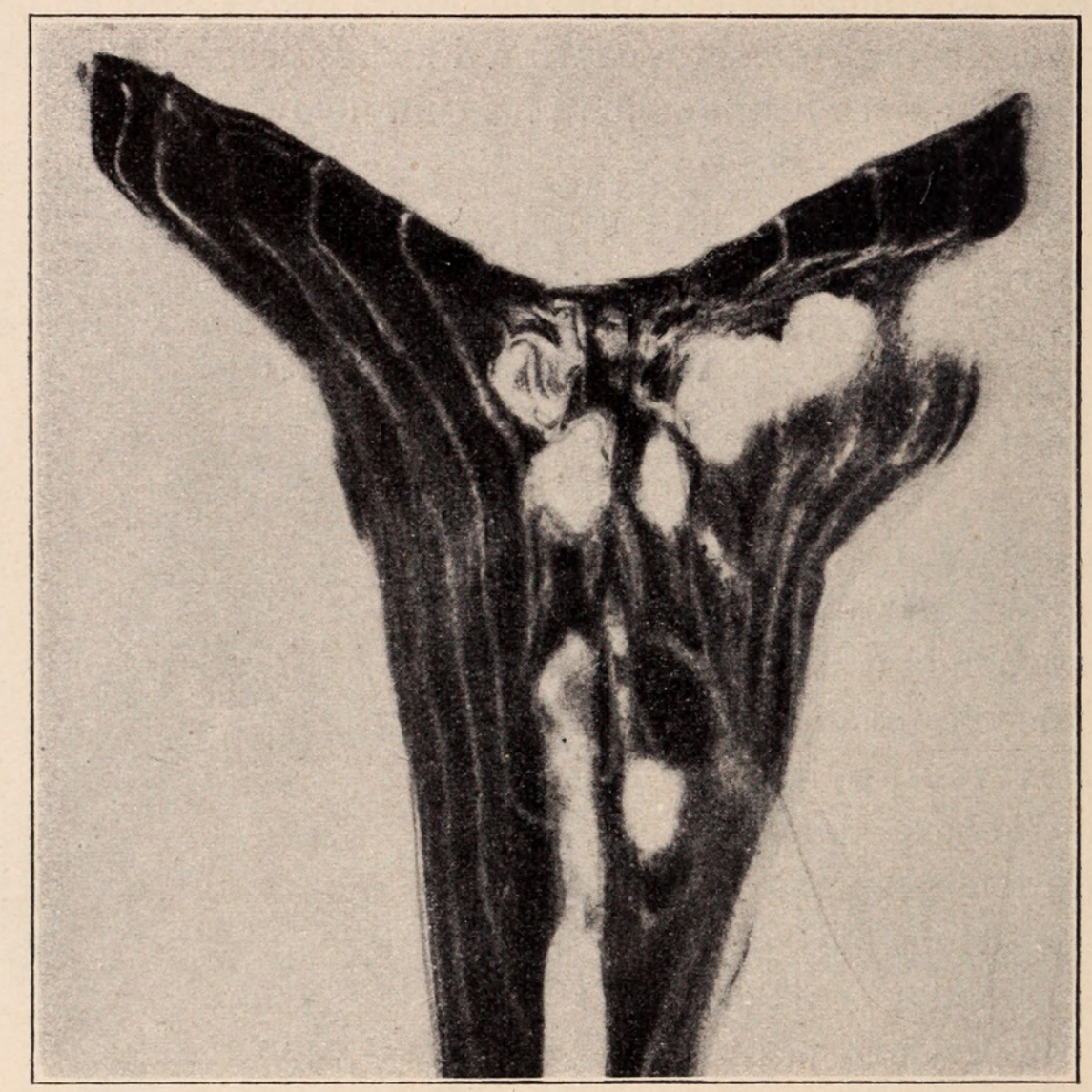

FIG. 6.

Dernière vertèbre du Carassin, coupe frontale.

Déjà EBNER (1875), et avant lui d'autres auteurs, ont observé l'influence des causes mécaniques sur la formation et la structure de l'os. Des expériences directes furent entreprises pour éclairer la portée de cette influence (par exemple l'extirpation des membres antérieurs chez des chiens nouveau-nés, etc.). On a recouru dans le même but à l'analyse des propriétés optiques de l'os examiné à la lumière polarisée, sans parler d'autres méthodes. Toutes ces recher- 
ches nous permettent de se faire une idée assez nette sur la dynamique de la croissance des os.

Différents auteurs prêtent une grande attention à la structure des os tubulaires nommée os concentriques "in toto". On comprend sous ce terme le parallélisme des systèmes longitudinaux de Hawers, la disposition concentrique de ces systèmes et de leurs ramifications. Geвнандт constate le fait suivant: le maximum de systèmes de Hawers longitudinaux et parallèles se trouve loin des points de fixation des grands tendons, la régularité de la disposition de ces systèmes est, d'autre part, influencée par les fonctions mécaniques de l'os. Mais ni Geвнавдт ni les auteurs plus récents n'ont su indiquer que cette disposition concentrique est en relation directe avec la croissance périostale de l'os.

J'ai soutenu au premier Congrès des zoologistes russes (1923), et antérieurement à Kazan (1916b), que les os des Mammifères y compris l'homme, malgré toute la complexité de la reconstruction interne dont ils deviennent le siège en avançant en âge, s'accroissent suivant une modalité identique à celle des Poissons et des Amphibiens. La complexité du processus de reconstruction jointe au peu d'intérêt qu'on a prêté à cette question ont seuls empêché jusqu'ici, de constater la signification réelle des lamelles basales externes, c'est-à-dire leur homologie avec les dépôts périostaux continus des Poissons et des Amphibiens.

La structure concentrique in toto provient, d'après ce que j'en peux juger par l'analyse des os humains, du fait que les voies de pénétration et de formation des canaux de Hawers sont dirigées suivant la ligne de moindre résistance. Celle-ci dépend de l'alternance de couches périostales de différentes densités à l'intérieur des os. Dans les régions où le processus de reconstruction est intense, les couches de lamelles basales ne se conservent que par place comme nous l'enseignent, d'ailleurs, les manuels d'histologie.

Avec l'âge, le processus de reconstruction peut avancer, au point que toute trace de couches périostales disparait; la structure concentrique des os peut, cependant, persister.

Etant arrivés à la conclusion que les dépôts périostaux sont homologues dans les différents groupes de Vertébrés (des Poissons jusqu'à l'Homme); nous devons en déduire que là où les phénomènes de résorption et de remplacement ont épargné par place ces dépôts, nous possédons des données sûres pourl'analyse de la périodi- 
cité de croissance. Si les couches périostales ne sont conservées que partiellement, il faut chercher dans le même os, ou bien dans d'autres os du même animal, des régions dont les dépôts sont synchrones, ceci tant en vue d'un contrôle que pour la reconstitution de la succession des couches.

La persistance dans la régularité de la structure concentrique in toto, indique que la direction générale des forces mécaniques change peu pour une région donnée d'un os durant la vie de l'animal. C'est à proximité de ces régions que l'on réussit d'ordinaire à trouver des séries plus ou moins continues de couches périostales.

La recherche d'endroits convenant à notre but présente certaines difficultés chez les Mammifères de grande taille, à cause de la complexité de la structure histologique de leurs os. Pour établir le synchronisme de grandes périodes de croissance, il faut parfois comparer entre elles non seulement des séries de lamelles osseuses apposées à la surface, mais aussi celles qui se trouvent à l'intérieur des cavités dues à une résorption antérieure.

Chez les Amphibiens et les Poissons, le processus de reconstruction est minime, c'est pourquoi tant qu'il s'agit d'élaborer une méthode d'étude de la périodicité de croissance, nous nous adressons de préférence à ces groupes de Vertébrés.

L'étude de la structure de l'os de nombreuses espèces de Poissons m'a convaincu que certains détails de celle-ci sont peu importants pour notre problème, telles sont l'absence ou la présence des corpuscules osseux, des systèmes de canaux osseux, etc. Nous observons chez des Poissons du même genre une grande variété dans les détails de la structure histologique de leurs os, tandis que le processus de l'apposition périostale suit son cours sans être influencé par les phénomènes causant cette différenciation. On peut parfaitement appliquer aux os de Poissons et d'autres Vertébrés le point de vue du professeur Zawarsin (1924) qui a si nettement exposé la théorie moderne sur le parallélisme de la structure morphogénétique adaptée aux éléments histologiques.

Pour l'élaboration de la méthode de l'étude de la croissance, la conception générale des processus morphogénétiques doit avoir un rôle décisif.

Quoique je sois loin de nier l'importance des actions mécaniques dans le processus de la croissance des os, puisque ma méthode porte le nom de "plans isodynamiques", j'ose affirmer que l'influence 
des agents externes n'est pas prépondérante dans le processus de la croissance de l'os. Ce rôle est dû à des causes plus profondes, telles que les particularités du plasma propres à une espèce donnée, l'autocatalyse dans la croissance et, en général, l'équilibre biochimique encore peu étudié. Comme la croissance dépend surtout de ces causes, il arrive que son intensité ne correspond pas au rôle mécanique effectif de l'os: un os très massif peut avoir des fonctions insignifiantes.

Les os ne sont pas l'unique exemple dans le règne animal, d'organes qui, au cours de l'évolution, acquièrent dans certains cas une grande massivité. Les paléontologistes indiquent maints exemples d'organes qui, étant devenus non seulement "inutiles " mais même " nuisibles », ont pu, pensent-ils, amener l'extinction de l'espèce (les coquilles de Mollusques, certaines formations chitineuses des Arthropodes, etc.).

Puisque les fonctions mécaniques jouent un grand rôle dans le processus de la reconstruction interne de l'os, il s'en suit que la périodicité de croissance sera visible surtout dans les os, ou dans les régions de ceux-ci, qui sont peu soumis à leur influence. L'os idéal pour notre étude serait celui qui, tout en étant massif ne subirait pas d'action tant soit peu intense de muscles ou de ligaments, et qui n'aurait pas à supporter le poids du corps. Chez les animaux aquatiques, ce dernier rôle est insignifiant; voici, peutêtre, la raison d'une uniformité relative dans la structure intime de leurs os.

Les otolithes s'approchent de l'idéal, car la direction de leurs plans de croissance dépend seulement, en dehors des causes-biochimiques, des forces qui se développent lors des mouvements de la tête.

Inversément, les os les moins propices à notre étude seront ceux qui, tout en étant pauvres en substance osseuse, ont d'importantes fonctions mécaniques.

Les plans convenables pour l'étude de la périodicité de croissance seront donc ceux où la direction et l'intensité relatives des forces se maintiennent sans - ou presque sanș - changements durant toute la vie de l'animal. Nous les dénommons pour cette raison: plans isodynamiques.

Dans la conception des plans isodynamiques, nous comprenons non seulement l'influence des causes biochimiques, mais aussi 
l'influence des forces mécaniques prises au sens strict de ce mot, telles: la pression sur l'os, la tension dans les courbures, la tension due à la rotation, le poids propre de l'os et les résultantes du déplacement de l'os dans l'espace.

Voici pour illustrer notre théorie quelques exemples concrets d'os, ou de régions d'os, convenant pour notre but. Je m'arrêterai principalement aux Poissons, car ce n'est que dans ce groupe que l'on a tenté une application étendue de cette méthode.

Certains rayons des nageoires dorsale et caudale des Cyprinidés sont très démonstratifs. Les fig. 7 et 8 représentent une coupe du

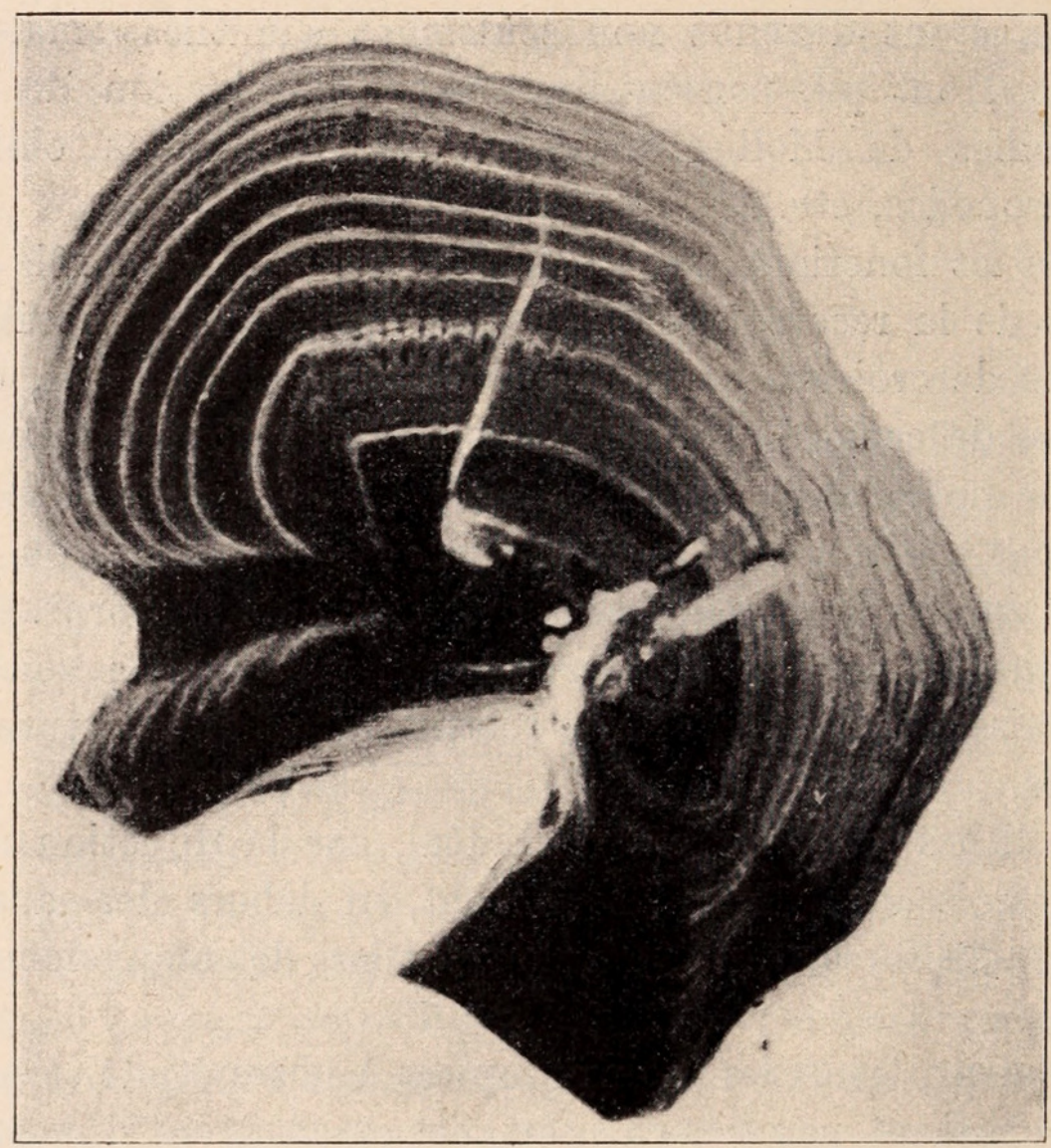

FIG. 7.

Quatrième rayon de la nageoire dorsale chez un Carassin, coupe transversale.

IV ${ }^{\text {me }}$ rayon de la nageoire dorsale ${ }^{1}$ du Carassin. Il est très massif, quoique ses fonctions soient insignifiantes, car il a pour fonction

${ }^{1}$ Le premier rayon étant très petit et dissimulé dans la peau, on prend souvent le IVme rayon pour le IIIme. 
de soutenir avec les autres rayons, une nageoire large et légère. Il y a bien à la base de ce rayon, à l'endroit où les vaisseaux sanguins pénètrent dans la gouttière intérieure de celui-ci, une cavité due à la résorption, mais une coupe juste au-dessus de celle-ci nous montre toute la série des dépôts périostaux.

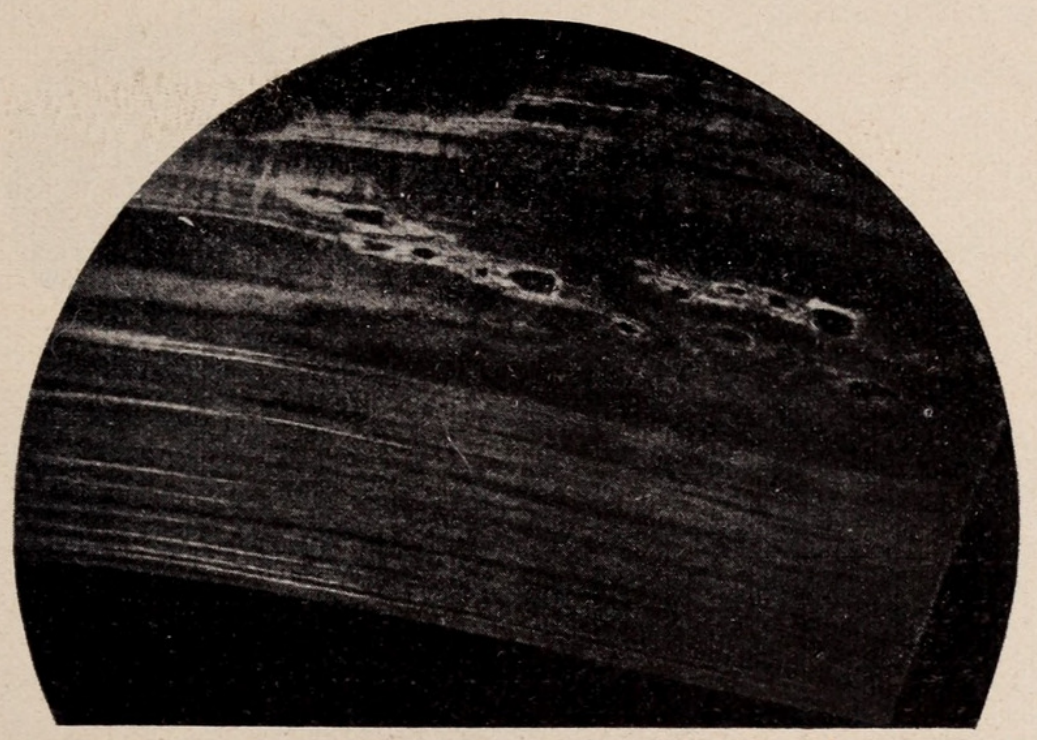

FIG. 8 .

Coupe longitudinale du mème rayon, lumière polarisée.

L'accroissement en longueur du rayon se fait par juxtaposition de tronçons, dont chacun est muni en arrière d'une saillie dentiforme. Ces tronçons sont cimentés ensuite par la substance osseuse qui se dépose uniformément à l'extérieur du rayon, de sorte que la surface postérieure reste longtemps dentelée, tandis que la surface antérieure est parfaitement unie. Cette égalité, ainsi que la persistance de la tension et la massivité des dépôts, permettent d'établir ici un plan convenable pour les sections qui s'orienteront perpendiculairement aux couches périostales.

Chez les Acipenseridae, on peut utiliser pour l'analyse beaucoup d'os, car ils sont presque tous massifs. Un des plus convenable sera l'os marginal de la nageoire pectorale ${ }^{1}$. La figure 9 représente la

${ }^{1}$ Dans un fascicule du Zool. Anzeiger (1924) parut une communication de Holzmayer, assistant au laboratoire de zoologie de Kazan, sur la détermination de l'âge du Sterlet d'après des coupes transversales de l'os marginal. M. HolzMAYER a omis de mentionner mon travail traitant le même sujet qui 
microphotographie d'une coupe faite à la base de celui-ci chez le Sterlet. On observe en lumière polarisée une succession de très minces lamelles superposées qui, par séries, correspondent plus ou moins aux cycles annuels de la croissance.

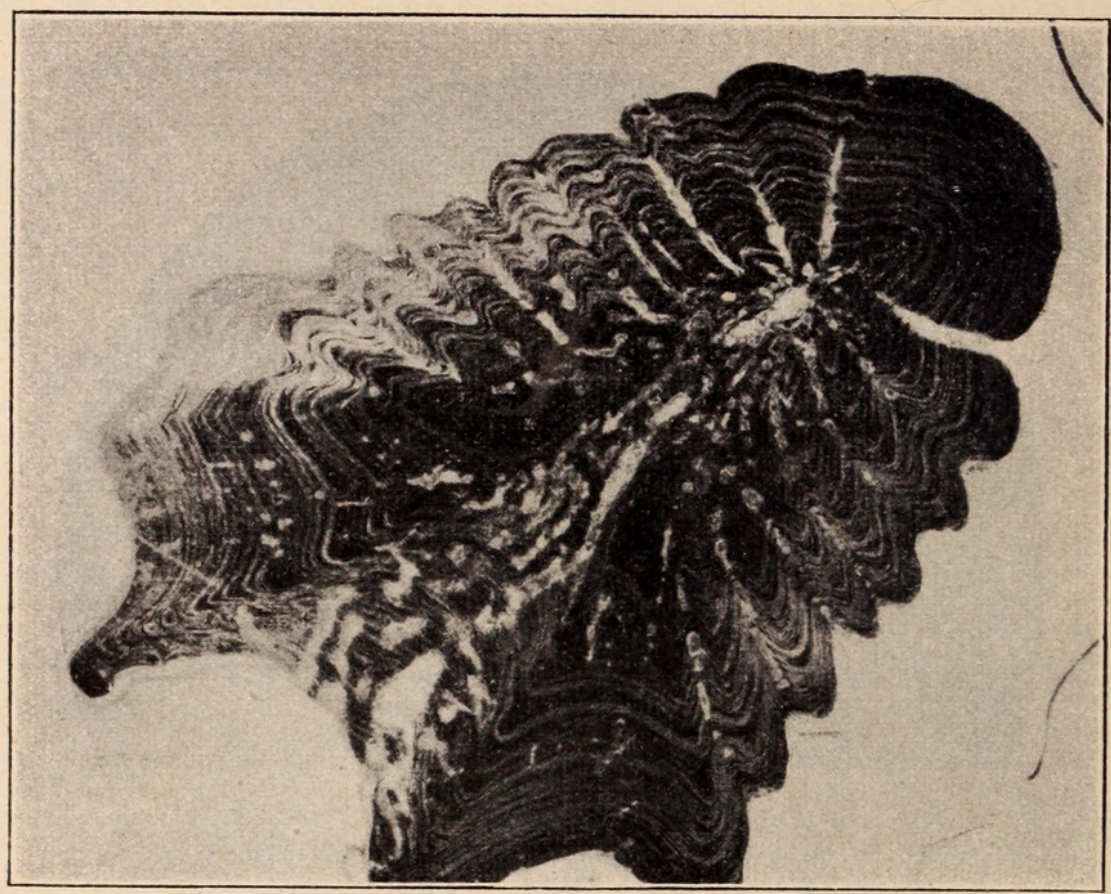

FIG. 9

Os marginal d'Acipenser ruthenus, coupe transversale très mince.

Une coupe d'un rayon d'un Huso huso pesant environ $800 \mathrm{~kg}$. montre que la résorption épargne suffisamment les dépôts correspondants aux premières années de vie même chez des Poissons très âgés (dans ce cas à peu près 50 ans). La massivité de l'os est ici loin de correspondre à l'importance de ses fonctions. Comme l'a démontré Schmalhausen, il sert partiellement de contre-poids au lobe dorso-caudal; il joue donc un rôle passif.

Chez le Sterlet (Acip. ruthenus) cet os tend à se tordre suivant

paru en 1916, de même que ma communication faite à la Société des Naturalistes de l'Université de Kazan (1916) et celle que j'ai faite en 1923 au Ier Congrès des zoologistes russes. Cette omission est d'autant plus étrange que M. HoLzMAYER, étant mon successeur dans le cabinet de zoologie, avait sous la main mes préparations originales, laissées là en dépôt. Je maintiens mon droit à la priorité dans l'élaboration de la méthode des coupes transversales pour l'étude de la croissance. 
l'axe longitudinal, mais cette tendance est suffisamment légère pour permettre d'orienter le plan de section perpendiculairement aux couches périostales, ceci dans la partie antérieure du rayon.

L'os maxillaire de plusieurs espèces de Salmonidae se prête bien à l'analyse de la croissance. La fig. 11 représente celui du Stenodus leucichtys nelma (Pall). L'excès de substance osseuse qui se dépose ici, en dehors de l'influence des muscles et tendons, est très grand, ce qui concourt à la formation des dépôts périostaux uniformément orientés.

La fonction de cet os comme on le voit sur la fig. 10 est de faciliter l'élargissement de la bouche. Lorsque celle-ci est fermée, l'os se

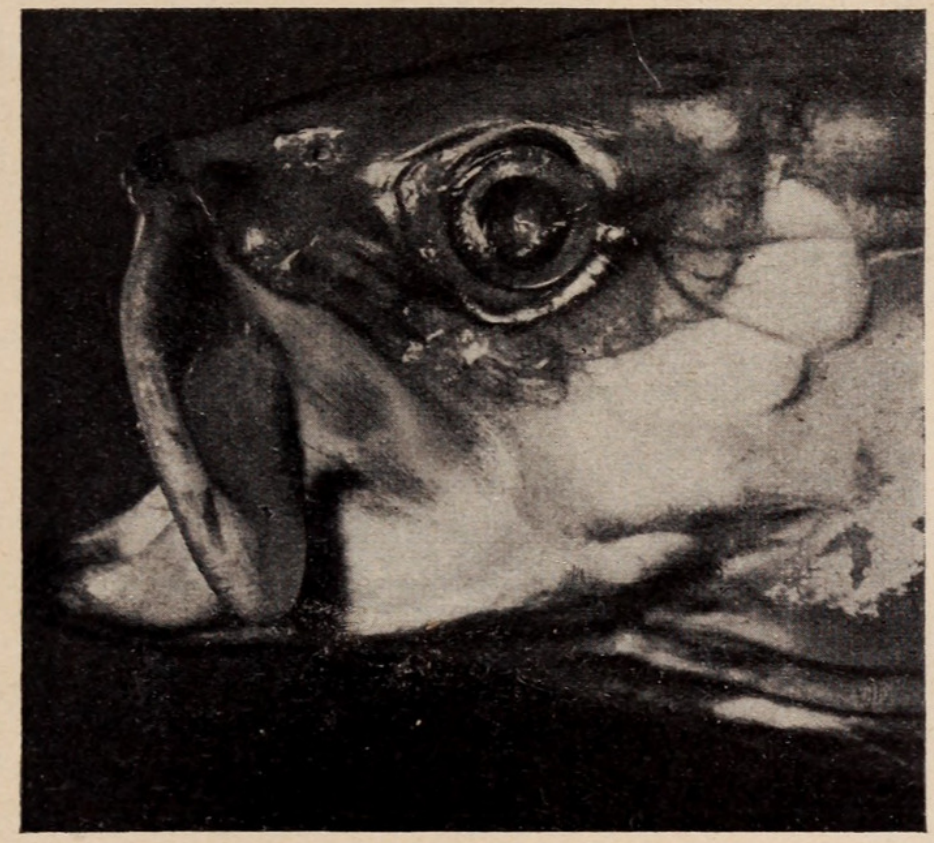

FIG. 10.

Tète de Stenodus leucichtys nelma, vue de côté L'os maxillaire est en position verticale.

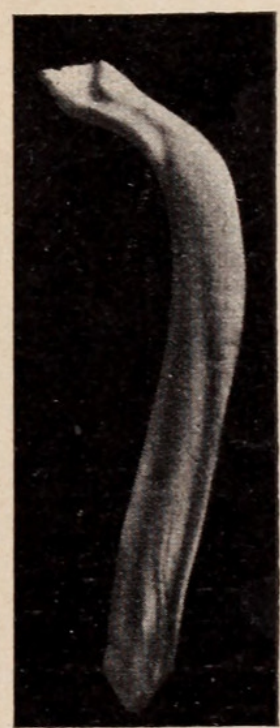

FIG 11.

L'os maxillaire de Stenodus leucichtys nelma,

vu de l'intérieur.

dispose presque horizontalement dans l'enfoncement correspondant; il est retenu là automatiquement par des faisceaux de tendons dont une partie est fixée au niveau de la branche ascendante dt l'os dental. Lorsque la bouche s'ouvre, l'os prend une position presque verticale sous la tension inverse des tendons et, en partie, sous la pression de l'extrémité antérieure de l'os palatin sur un tubercule situé près de l'articulation. Cette pression fait tourner l'os d'un 
certain' angle suivant son axe longitudinal et contribue à l'élargissement de la bouche.

Un os mince et plat pourrait facilement remplir, - et, de fait, remplit - ces mêmes fonctions chez d'autres Poissons.

Nous trouverons facilement un plan isodynamique en faisant une section perpendiculaire à l'épaississement antéro-postérieur du col de l'os. Il est possible d'orienter ici la section de façon à pouvoir mesurer l'épaisseur relative des couches périostales (fig. 12). Il va sans dire que ce "plan isodynamique" ici, comme ailleurs, n'est en réalité que la partie d'une surface sphérique. Dans le

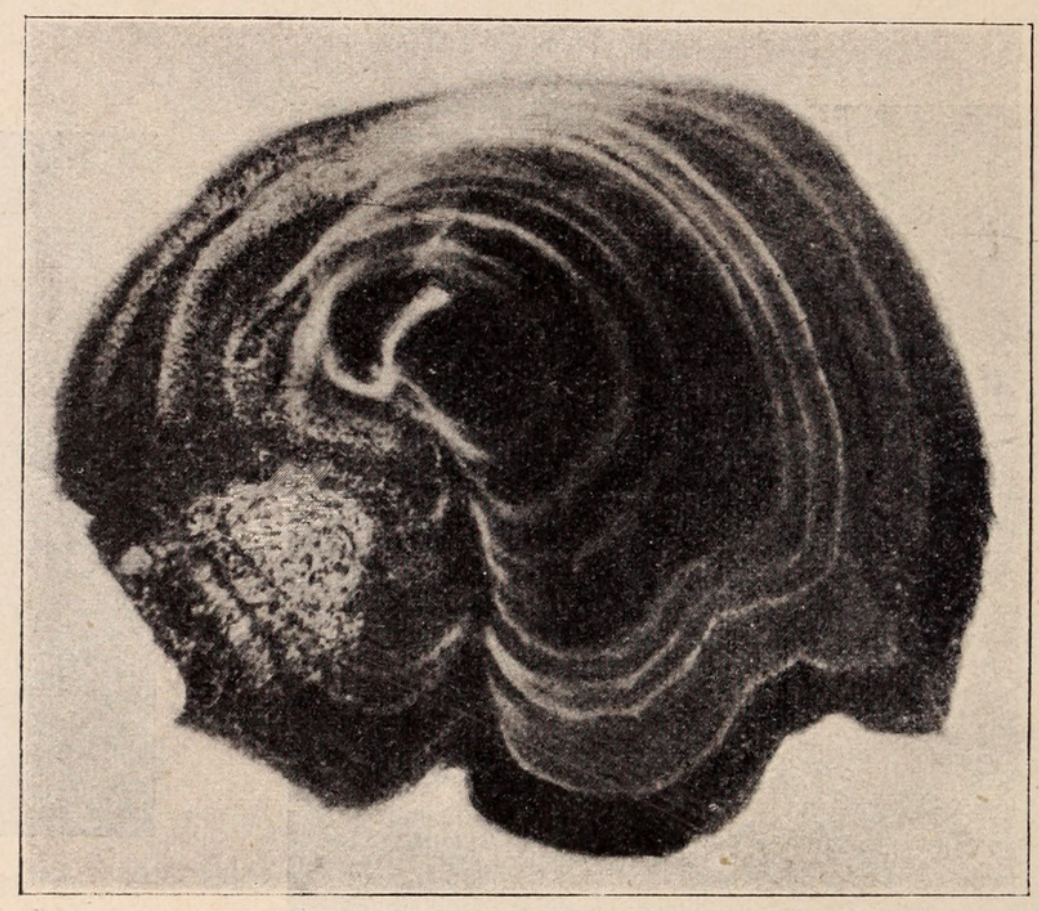

FIG. 12

Coupe transversale du col de l'os maxillaire chez le Stenodus leucichtys nelma.

quadrant indiqué, la courbure est si faible qu'elle permet d'orienter la section perpendiculairement aux couches périostales, ce qui est suffisant pour l'analyse; les autres quadrants ont une courbure plus prononcée.

J'ai indiqué plus haut que les os plats sont le siège d'une résorption interne très prononcée. Il est, néanmoins, aisé de trouver ici des plans isodynamiques si l'on tient compte de la présence dans 
beaucoup d'os de parties massives, telles que les excroissances. Dans les ornements, où les forces mécaniques ne sont pas en jeu, la croissance végétative se manifeste par des dépôts de substance osseuse ou ostéoïde suffisamment continus.

L'os préoperculaire de la Perche peut nous servir d'exemple (voir fig. 13). On voit la structure d'une crête située le long du milieu de l'os. La résorption, très intense dans ce dernier os, épargne la crête qui est neutre au point de vue des fonctions mécaniques.

Comme il a été déjà question de la structure des vertèbres je n'y reviendrai plus et me bornerai à indiquer l'importance de ces os pour l'étude des Poissons fossiles et sub-fossiles. La méthode des plans isodynamiques donne ici des résultats très satisfaisants. L'essentiel est de savoir trouver une orientation convenable pour les sections (fig. 4, 5). Quoique la structure des vertèbres soit très variée, il est toujours possible de trouver dans la direction radiale un plan perpendiculaire aux couches périostales. Chez bien des Poissorns c'est la dernière vertèbre qui se prête le mieux à l'étude, car les dépôts périostaux sont ici très puissants sur la surface amphicoele, ce qui facilite l'orientation du plan de section (fig. 6).

La clavicule (fig. 14) du Huso huso présente un exemple très démonstratif de résorption superficielle. Le bord externe de cet os est massif et

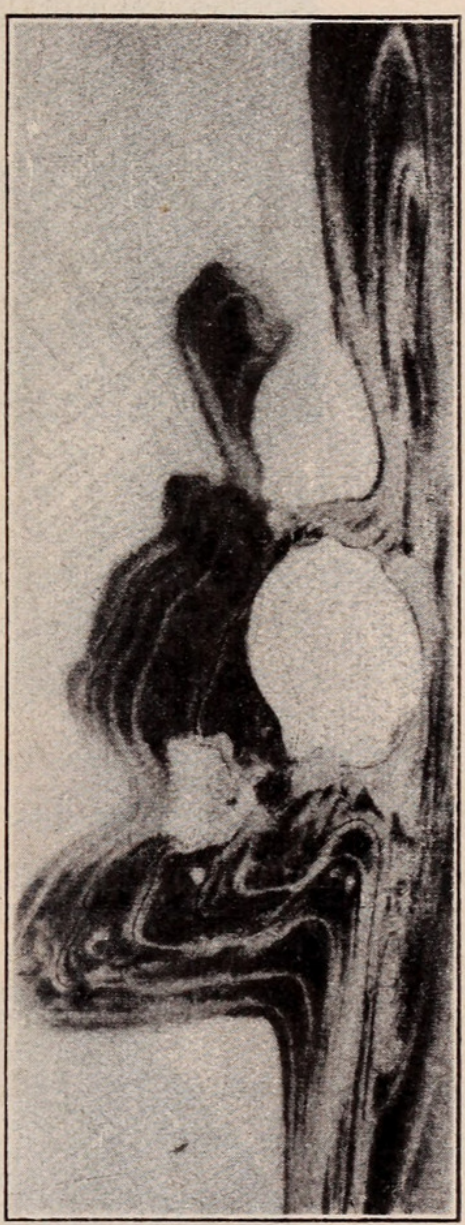

FIG. 13.

Os préoperculaire de la Perche. Coupe à travers la crête longitudinale. muni d'épaississements en forme de crêtes disposées en éventail. Vers le centre, l'os s'amincit jusqu'à former des trous dus à la résorption superficielle. Cette dernière aplanit aussi les crêtes au fur et à mesure de la croissance. Cependant, on trouve sur le même os une région épargnée par la résorption, à savoir le point central de la courbure. 
En ce qui concerne les Mammifères, l'application de ma méthode est surtout démonstrative sur les os humains, où le processus de

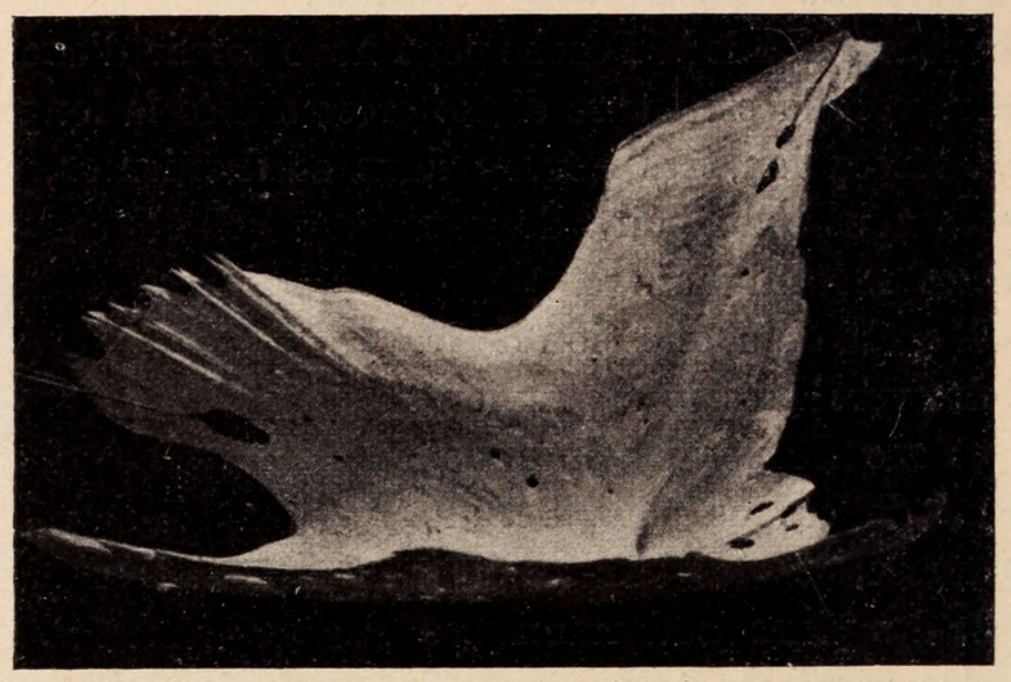

FIG. 14.

Clavicule de Huso huso. On voit les crêtes sur le bord extérieur et un amincissement près du centre.

reconstruction est très complexe. La microphotographie 15 donne une idée du caractère des dépôts périostaux de la squama temporalis vue en lumière polarisée; il est possible de suivre ici les séries de

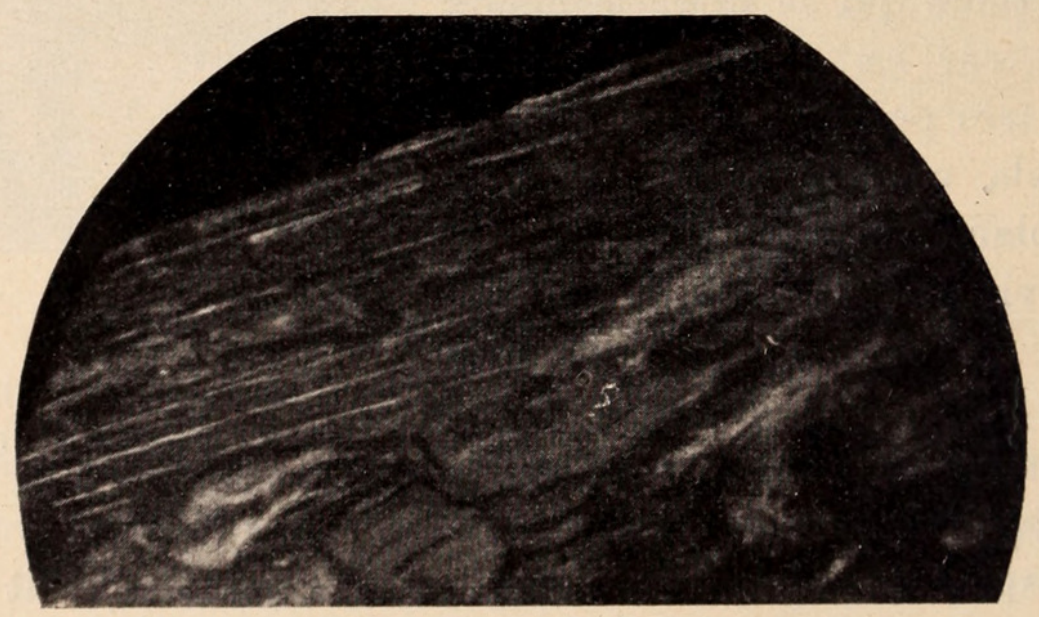

FIG. 15.

Coupe à travers l'os temporal (squama temporalis) d'un Homme âgé de 40 ans (Lumière polarisée.) 
couches correspondant à de longues périodes de croissance. Nous trouvons également des régions analogues dans d'autres os de la voûte cranienne.

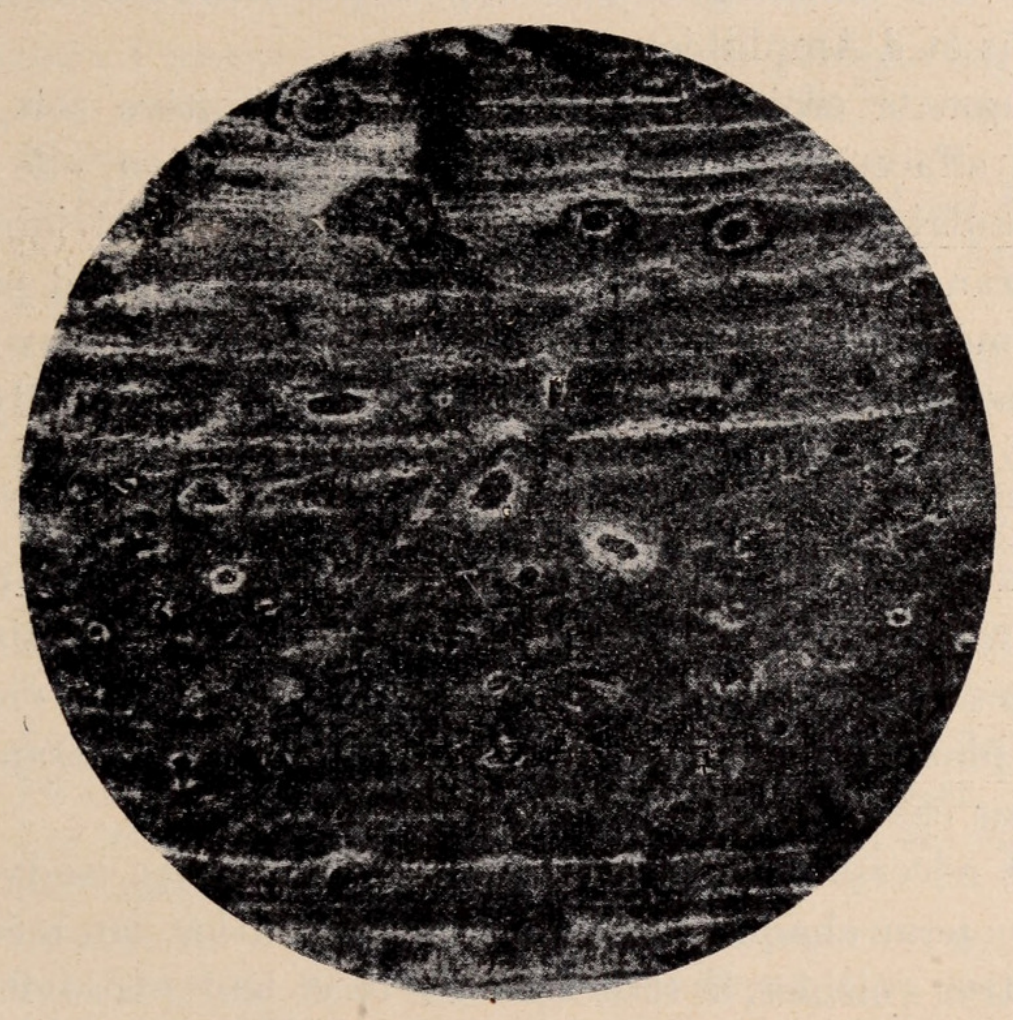

FIG. 16

Lamelles basales internếs et externes; fémur d'un garçon de 8 ans; milieu de lá diaphyse (d'après Loviaguine).

La microphotographie 16, empruntée au travail de Loviaguine (1915), représente une coupe à travers le milieu de la diaphyse du fémur chez un garçon de 8 ans.

\section{Conclusions.}

1. Les méthodes employées dans l'ichtyologie pratique pour la détermination de l'âge et des périodes de frai des Poissons, basées seulement sur l'étude de l'écaille, des vertèbres ou des os plats, ne suffisent pas pour l'étude détaillée des différentes phases de la périodicité de croissance. 
2. Le dépôt périostal de la substance osseuse joue chez les Vertébrés un rôle très important dans le processus de croissance et de formation de l'os. Les lamelles basales externes des Vertébrés supérieurs sont homologues aux dépôts périostaux continus des os de Poissons et d'Amphibiens.

3. Quoique la croissance concentrique primitive soit, chez les Vertébrés, effacée de bonne heure par la résorption, elle est cause de la concentricité de la structure des os dits concentriques in toto.

4. La grande massivité de certains os ne correspond pas à leurs fonctions mécaniques. Dans ce cas, les dépôts périostaux sont tout particulièrement réguliers et démonstratifs pour l'analyse de la périodicité de croissance.

5. Les forces mécaniques ont une influence sensible sur la reconstruction interne de l'os. Il en résulte que les os - ou les régions de ceux-ci où la relation entre les forces ainsi que leur direction se maintiennent dans les mêmes plans durant toute la vie - conviennent plus spécialement à l'analyse de la croissance. Nous dénommons ces plans: "plans isodynamiques".

6. Il est nécessaire dans la recherche des plans isodynamiques d'examiner pour chaque espèce le squelette entier, car, même quand elles sont très voisines, la structure des os et leur massivité relative varient beaucoup.

7. Chez l'Homme et les autres Mammifères, les os de la voûte cranienne, ainsi que les régions des os tubulaires où les points d'application de la force musculaire font défaut, se recommandent pour l'étude de la périodicité de croissance.

Laboratoire de Zoologie de l'Institut Polytechnique à Sverdlovsk (Ekatherinbourg). 


\section{INDEX BIBLIOGRAPHIQUE ${ }^{1}$}

1924. Ancona Umberto, d'. - Contributo alle Biologia degli Storioni nelle aque italiene. Ministere d'Economia Nazionale, Div. 5 , 1924.

1890. Axel-Kay, L. - Die Pubertätsentwicklung und das Verhältniss derselben zu den Krankheitserscheinungen der Schuljugend. Int. Med. Congr., Bd. I, Berlin, 1890.

1924. Benninghofi, A. - Experimentelle Untersuchung über den Einfluss verschiedenartiger mechanischer Beanspruchung auf den Bau der Knorpel. Anat. Anz. 1924.

1916a. Clenc. W. - Quelques données sur la définition de l'âge des Poissons d'après les os. Wiestnik Ribopromischlennosti, Petrograd, 1916, No 5.

1916 b. - La définition de l'àge des Poissons d'après les os. C. R. de la Soc. Nat. de l'Université de Kazan, No 499, 1916 (en russe).

1923. - La périodicité de croissance des os et leur structure chez l' Homme. Ier Congrès des zoologistes russes, 1923 (en russe).

1924. - Les Poissons nains. II Congrès des zoologistes russes, 1924 (en russe).

1920. DuERSt, J. H. --Vergleichende Untersuchungsmethoden am Skelett bei Säugern. Abderhalden's Handbuch biol. Arbeitsmeth. Lief. 200, 1920.

1922. Dubreuil, G. - Les phénomènes de résorption dans les os de la voûte cranienne. C. R. Ass. Anat., 1922.

1875. Ebner, V. v. - Ueber den feineren Bau der Knochensubstanz. Sitz. d. K. K. Akad. der Wiss. in Wien, 1875.

1882. - Untersuchungen über die Anisotropie organisierter Substanzen. Leipzig, 1882.

1922. Gandolfi, H. A. - Recherches sur l'àge et la croissance de quelques Anguilles argentées. Ann. Biol. lacustre, 1922.

1900-05. Geвнавdт, W. - Ueber funktionell wichtige Anordnungsweisen der feineren und gröberen Bauelemente der Wirbeltierknochen. Arch. f. Entw.-Mech. 1900, 1901 und 1905.

1915. Foote, M. D. - A contribution to the comparative Histology of the femur. Washington. Smiths. Inst., No 2382, 1915.

1881. Kastchenko, N. - Ueber die Genese und Architektur der Batrachierknochen. Arch. f. wiss. Anat., 1881.

1924. Кондrausch, W. - Ueber den Einfluss funktioneller Beanspruchung auf das Längenwachstum von Knochen. Münch. med. Woche, 1924.

1 J'omets de mentionner les nombreux travaux suffisamment connus concernant la détermination de l'âge des Poissons. 
1858. KöLliker, A. -- Ueber verschiedene Typen in der mikrostcopischen Struktur des Skelettes der Knochenfische. Würzb. Verhandl., Bd. 9, 1859 .

1889. - Handbuch der Gewebelehre, 1889.

1926. Kotikov, E. - Recherches sur les causes qui ont une influence sur la forme du tibia. Bull. de l'Inst. Leshaft, 1926. (En russe).

1924. Lillie, R. - The physico-chemical conditions of Morphogenesis. Amer. Natural. 1924.

1915. Loviaguine, N. - Matériaux concernant la structure microscopique des os. Dissert. (en russe), 1915.

1925. MaAs, H. - Knochenwachstum und Knochenaufbau. Virch. Arch., 1925.

1921. - Kritische Studie zur Physiologie und Pathologie des Knochenwachstums. Stuttgart, 1921.

1891. Matschinsky, N. - Croissance normale des os tubulaires de l'homme. Dissert. med. (en russe), 1891.

1904. Mey iu Rg, H. - Beitrag zur Kenntnis des Stadiums der primären "in toto" konzentrischen Knochenbildung. Arch. f. mikr. Anat. und Entw. 1904.

1909. Nowikov, M. - Recherches sur les tissus cartilagineux et osseux. Ann. scient. de l'Univ. de Moscou, 1909. (En russe).

1914. Ostrooumov, A. A. - Das dritte Jahr des Wachstums des Sterlets. Bull. de la Soc. Nat. Kazan, t. XLVI (en russe), 1914.

1912. Pouchet, R. - Développement du squelette des Poissons osseux. Journal de l'Anat. et de la Phys. XI, XII, 1912.

1922. Quix, P. H. - Les fonctions des otolithes. Arch. néerl. Physiol., 1922.

1908. Retterer, E. - Influence de l'activité ou du repos sur la structure des tissus osseux. Compte rendu de l'Ass. Anat., 1908.

1883. Schmidt-Monnard, C. - Die Histogenese des Knochens der Teleostier. Zeitschr. f. wiss. Zoologie. Bd. 39, 1883.

1898 Stephan, E. - Sur les cellules propres à la substance osteoüde des Poissons téléostéens. C. R. Soc. Biol. 1898.

1898. Stratz, E. H. - Naturgeschichte des Menschen. Stuttgart, 1898.

1923. Lorou R, M. F. - Versuche über Einfluss von Nahrung, Licht und Bewvegung auf Knochenentwicklung. Ziegl. Ber. path. Anat., 1923.

1922. Weidenreich, F. - Ueber die Beziehungen zwischen Muskelapparat und Knochen und den Charaliter des Knochengexvebes. Virch. Anat. Gest., 1922.

1924. Zawarsin, A. - Der Parallelismus der Struktur als ein Grundprinzip der Morphologie. Zeit. f. wiss. Zool., 1924. 


\section{$2 \mathrm{BHL}$ Biodiversity Heritage Library}

Clerc, W. 1927. "Etude de la périodicité de croissance d'après les plans isodynamiques des os." Revue suisse de zoologie 34, 477-498. https://doi.org/10.5962/bhl.part.117613.

View This Item Online: https://www.biodiversitylibrary.org/item/148557

DOI: https://doi.org/10.5962/bhl.part.117613

Permalink: https://www.biodiversitylibrary.org/partpdf/117613

\section{Holding Institution}

American Museum of Natural History Library

\section{Sponsored by}

BHL-SIL-FEDLINK

\section{Copyright \& Reuse}

Copyright Status: In copyright. Digitized with the permission of the rights holder.

Rights Holder: Muséum d'histoire naturelle - Ville de Genève

This document was created from content at the Biodiversity Heritage Library, the world's largest open access digital library for biodiversity literature and archives. Visit BHL at https://www.biodiversitylibrary.org. 